Repository of the Max Delbrück Center for Molecular Medicine (MDC) Berlin (Germany)

http://edoc.mdc-berlin.de/9233/

\title{
Cerebral blood perfusion changes in multiple sclerosis
}

Jens Wuerfel, Friedemann Paul, and Frauke Zipp 


\title{
Cerebral blood perfusion changes in multiple sclerosis
}

\author{
Jens Wuerfel ${ }^{1}$, Friedemann Paul ${ }^{1}$, and Frauke Zipp ${ }^{1}$ \\ ${ }^{1}$ Institute of Neuroimmunology, Charité — Universitätsmedizin Berlin, Germany
}

\begin{abstract}
The proximity of immune cell aggregations to the vasculature is a hallmark of multiple sclerosis. Furthermore, it is widely accepted that inflammation is able to modulate the microcirculation. Until recently, the detection of cerebral blood perfusion changes was technically challenging, and perfusion studies in multiple sclerosis patients yielded contradictory results. However, new developments in fast magnetic resonance imaging have enabled us to image the cerebral hemodynamics based on the dynamic tracking of a bolus of paramagnetic contrast agents (dynamic susceptibility contrast). This review discusses the technical principles, possible pitfalls, and potential for absolute quantification of cerebral blood volume and flow in a clinical setting. It also outlines recent findings on inflammation associated perfusion changes, which are inseparable from pathological considerations in multiple sclerosis.
\end{abstract}

KEYWORDS I Perfusion; Magnetic Resonance Imaging; Multiple Sclerosis; Inflammation; Blood-brain Barrier; Dynamic Susceptibility Contrast.

\section{Introduction}

One of the crucial stages in the evolution of a multiple sclerosis lesion is considered to be the disruption of the blood brain barrier (BBB) $[1,2]$, leading to edema in the CNS by accumulation of plasma fluids. This process is believed to be initiated by autoreactive CD4+ lymphocytes which migrate into the CNS and start an inflammatory response. Although BBB breakdown imaged as focal enhancement in T1- weightedMRI after gadolinium-DTPA(Gd-DTPA) injection - is the gold standard of lesion detection during the course of the disease, the deposition of contrast agent in the CNS has been shown to correlate only modestly with clinical disability. However, new imaging techniques, which allow more accuracy inmonitoring the disease evolution, provide evidence of tissue damage in the normal appearing white matter (NAWM) preceding the appearance of newcontrast-enhancing lesions. A decrease in magnetization transfer ratio (MTR) is described prior to enhancement, indicating a diminished ability for saturation exchange due to edema and inflammation $[3,4]$. Changes in the lipid spectra have been noted in magnetic resonance spectroscopy preceding lesions [5]. Similarly, subtle increases in the random water molecule motion reflecting alterations in tissue integrity were detected weeks before leakage of the BBB by means of diffusion weighted imaging [6].

However, local changes in blood flow, a major consequence of inflammation [7-9], have so far received little attention. Perfusion imaging has been performed in several other neurological diseases, such as stroke and different forms of dementia. Here, reduced perfusion parameters in several brain regions were linked to decreased metabolic activity secondary to neurodegeneration $[10,11]$. Although in multiple sclerosis only few perfusion studies have been published so far, findings of very early focal perfusion changes during the inflammatory phase of the disease [12], and of generally reduced grey matter perfusion in MSpatients compared to healthy controls [13] might indeed influence our understanding of the underlying disease processes, and could be important for future therapeutic considerations.

\section{In vivo perfusion measurements: Technical background and pitfalls}

Cerebral perfusion is defined as the volume of blood flowing through a given volume of tissue per unit of time [14]. It is comprised of three parameters: the cerebral blood flow (CBF), the cerebral blood volume (CBV), and the mean transit time (MTT). Although the first reports on human cerebral flow measurements using magnetic resonance imaging date back as far as 1970 [15], perfusion measurements have remained technically challenging. In 1988, a transient change in contrast after injection of lanthanide chelates (Gd-DTPA) into healthy rats was described and explained by susceptibility effects in the microenvironment [16]. Such microscopic gradients lead to fast dephasing of proton spins, and hence to a reduction in the transverse relaxation time (a decrease in T2/T2*), which can be detected as signal loss during the fast passage of a contrast agent through the tissue (first pass effect). This effect dominates over the simultaneous T1 relaxation enhancement and extends well beyond the intravascular compartment; it also affects the surrounding tissue even though nondiffusible tracers, such as GdDTPA, remain strictly intravascular in the presence of an intact BBB [17].

Imaging the first pass effect of intravenously injected paramagnetic contrast agents with high temporal resolution is called Bolus Tracking or Dynamic Susceptibility Contrast Magnetic Resonance Imaging (DSC-MRI). It is the most commonly used method to investigate hemodynamic changes. Maps of CBV, CBF and MTT are calculated on the assumption of an approximately linear relationship between the concentration of the contrast agent in the tissue and the change in $\mathrm{T}^{*}$ relaxation rate. CBV is determined by integrating the tissue concentration time curve of each voxel, and CBF by the deconvolution of this curve with an arterial input function (AIF: the concentration of contrast agent in the feeding vessel to the volume of interest (VOI) at a given time) [18]. MTT, the average time required for a given particle of tracer to pass through the tissue, can be calculated by the central volume theorem MTT=CBV/CBF [19]. Due to the rapid bolus transit, fast imaging is required in order to capture the first pass of the bolus (e.g. repetition time (TR) b1.5 s). Rapid injection of the contrast agent followed by a saline flush is imperative to obtain a sharp input bolus to the tissue (e.g. N3 ml/s). Today, 
the availability of fast imaging techniques such as epiplanar imaging (EPI) allows us to obtain sequential images of the wash in and wash out phase of a tracer during the few seconds of bolus transit through the tissue. Until recently, its use in clinical studies was restricted due to a number of technical limitations, such as a relatively poor spatial resolution leading to partial volume effects [20], limited volume coverage, and a low signal-to-noise ratio (SNR). Increased gradient performance and higher field strengths made perfusion MRI feasible even in a routine clinical setting. However, some caveats should be taken into consideration. Since the tracer concentration cannot be measured directly by MRI, it must be measured indirectly through its effect on signal intensity. Therefore the concentration of the contrast agent in various tissue compartments must be known or estimated in order to derive hemodynamic parameters from dynamic tracer analyses. As vascular structure has a strong influence on flow, an accurate acquisition of the AIF is essential [21]. The AIF depends not only on the shape of the injected bolus but also on the cardiac output, the vascular geometry, and the cerebral vascular resistance $[22,23]$. Accordingly, the site of the AIF estimation should be chosen as close to the region of interest as possible. For the quantification of absolute CBF, the AIF must be deconvolved. Several model-dependent and modelindependent deconvolution methods have been assessed and discussed in the past [24-28], and were recently reviewed by Ostergaard [18]. The selection of an approach sufficiently reflecting the in vivo situation is necessary for the correct prediction of absolute CBF values [29].

In some pathologies, such as multiple sclerosis, a conspicuous artefact can be observed in case of permeable capillary walls due to the defective BBB, e.g. in contrast-enhancing lesions. Here, the above mentioned assumption of a negligible effect of the contrast agent influx on T1 do not hold true. Leakage into the tissue leads to an artificially low estimate of CBV. Haselhorst et al. described an algorithm which can be applied to correct for the leakage artefact by fitting a model function for the plasma concentration to the measured data [30].

\section{Cerebral perfusion changes precede blood-brain barrier breakdown in MS}

Early measurements of global perfusion in multiple sclerosis using radioactive ${ }^{133} \mathrm{Xe}$ [31], positron emission tomography (PET) [32], or single photon emission computed tomography (SPECT) techniques [33] suffered from a very low spatial resolution. Findings of a generally lower perfusion in MS patients compared to healthy individuals were contradicted by the demonstration of increased perfusion during the time of acute inflammation in an animal model of MS [34].

With the advent of modern MR imaging and postprocessing techniques, it became possible to differentiate alterations in cerebral perfusion more accurately. Rashid et al. reported a hypoperfusion in the grey matter tissue, particularly in the thalamus and the caudate nuclei, and an increase in white matter perfusion in comparison to healthy controls, applying a novel continuous arterial spin labelling (CASL) technique and a statistical parametric mapping analysis (SPM) [13]. Haselhorst et al. were among the first to investigate different MS lesion types in a cross-sectional study [30].
After application of an extended BBB leakage correction to the data, the authors found a significantly higher CBV in acute Gd-DTPA enhancing plaques and a lower CBV in T1 hypointense lesions in comparison to the NAWMand T1 isointense tissue. Similar results were shown in a study by $\mathrm{Ge}$ et al. [35]. Our own group presented the first longitudinal data on perfusion changes during the genesis of MS lesions [12]. We confirmed that acute plaques are increased in both $\mathrm{CBF}$ and $\mathrm{CBV}$ prior to contrast enhancement, and that decreased perfusion persists in those plaques that remained T1 hypointense after several weeks. To our surprise, hyperperfusion developed weeks before lesions became visible in T2 or T1 weighted images, and even before changes in diffusion measurements were detectable. This was the first in vivo evidence that alterations in cerebral perfusion occur very early during the development of MS lesions, preceding overt changes in BBB permeability, T2 relaxation and diffusion properties. Recently, Broom et al. published a study which confirmed our findings in a rodent model of multiple sclerosis (experimental autoimmune encephalomyelitis, EAE) [36]. By investigating an animal model, his group was able to correlate perfusion and diffusion MRI findings with histopathology. Interestingly, regions with increased perfusion before contrast enhancement corresponded to areas with vessel dilatation, but could not be correlated to marked leukocyte infiltration, which defines an early stage of the inflammatory response with very low levels of macrophages, TandB cells.Up to date, technical limitations restricted the minimal size of a lesion to be followed (e.g. 5- $7 \mathrm{~mm}$ ) [12]. Future improvement in resolution may enable us to differentiate even smaller plaques and to predict their development based on the regional hyperperfusion. Thus, perfusion MRI might become a more sensitive tool for detecting the onset of inflammatory changes in MS than any other conventionally used MRI parameter.

\section{Pathophysiological considerations}

The close vicinity of evolving plaques to venules demonstrated histologically in human brain tissue [37,38], by MRI [39] and in animal models [40,41] - in MS suggests that perfusion and inflammation share common pathophysiological mechanisms. However, their relationship is far from being completely understood. Research in this area has focused both on vasoactive properties of soluble substances / cytokines produced and released during inflammation and on changes in the cellular homeostasis in astrocytes. Altered endothelial cell function might be the common final pathway leading to changes in perfusion and BBB leakage.

In one of the first perfusion studies in MS, changes in the blood supply during inflammation were attributed to a heightened glucose metabolism resulting from increased inflammatory cell activation [34]. Effective vasodilators such as nitric oxide (NO) and substance $P(\mathrm{SP})$ presumably originate during brain inflammation $[42,43]$. SP-producing immune reactive cells were identified at the edge of both active and inactive MS lesions, suggesting that substance $\mathrm{P}$ may play a role in $\mathrm{MS}$ lesion development [42]. More recently, the effects of primary inflammatory cytokines were studied in several models of neuroinflammation. An increase in the permeability of the BBB and higher CBV were seen after intrathecal application of interleukin-1 $\beta$ [44]. On the other hand, a 
reduction of the CBV was observed in response to direct intrastriatal injection of tumour necrosis factor- $\alpha$ (TNF $\alpha$ ), possibly reflecting a later stage of inflammation in this model [45]. TNFa and interferon- $\gamma$ (IFN $\gamma$ ) are known to rapidly induce cultured endothelial cells to synthesize tetrahydrobiopterin, a limiting cofactor for the endothelial $\mathrm{NO}$ synthetase (eNOS) converting L-arginine to $\mathrm{NO}$ and Lcitrullin, thereby increasing NO production by activating eNOS [46]. Only at later stages does TNF $\alpha$ diminish NO release by destabilizing eNOS transcripts [47] and by reducing protein kinase $B$ mediated phosphorylation of eNOS [48]. Production of NO by the inducible NO synthetase (iNOS) has also been implicated in the pathogenesis of MS. In a rodent model of $E A E$, the expression of iNOS in the CNS correlated with the severity of the disease, which was reversed by treatment with antisense cDNA [49] or NO scavengers [50]. Expression of iNOS has been found in active demyelinating lesions of postmortem brain tissue [51]. Interestingly, elevated levels of NO, TNF $\alpha$ and interleukin10 were also described in the cerebral spinal fluid of MS patients [52].

In addition to NO and cytokine mediated changes in cerebral perfusion, disturbed glial cell homeostasis may contribute to a heightened susceptibility for vascular changes in MS patients. Recently, it was reported that a rise of calcium levels within astrocytes induces constriction of blood vessels and consequently reduces cerebral blood flow [53]. The absence or reduction of astrocytic factors causes BBB endothelial cells to carry a reduced number of mitochondria. $A$ decreased mitochondrial content in endothelial cells in MS postmortem tissue is hypothesized to be a primary event in the disruption of the BBB [54].

On the other hand, early vascular endothelial cell activation was identified prior to cerebral parenchymal reaction and demyelination [55], which, in combination with discrete microglial activation and perivascular cellular infiltrates, is a frequent finding in postmortem tissue of MS patients [56]. Microglial activation coincided with abnormal endothelial tight junctions in active lesions and normal appearing white matter, leading to putatively open junctions in microscopically inflamed vessels [57]. Future studies will have to prove whether and which vascular factors in MS patients may facilitate the inflammatory process, and whether vascular vulnerability in our patients is a prerequisite for the initiation of the autoimmune cascade.

\section{Conclusion}

In recent years, MR imaging has gained increasing importance in the diagnosis and monitoring of disease activity in multiple sclerosis. While the correlates of a BBB disruption revealed by conventional imaging techniques are relatively well understood, little is known about the potentially even more devastating early and diffuse processes during the disease course. Alterations in tissue integrity, axonal loss and neuronal damage take place in the normal appearing white and grey brain matter, so far undetectable by conventional MRI techniques. Recent studies using Diffusion MRI (DTI), Magnetization Transfer Imaging (MTI), or Proton-Spectroscopy (HMRS) demonstrated that some of these changes are detectable already in the earliest phase of the disease, the clinical isolated syndrome, and that these precede the plaque development visualized by conventional MRI. Only a few studies have so far investigated the alterations of cerebral blood perfusion caused by inflammation. This might be due to technical difficulties in the past, which hindered sufficiently high spatial and temporal resolution, though these difficulties can be overcame today. Very recently it was shown that each inflammatory stage presents its own perfusion characteristics, e.g. hyperperfusion in the acute stage of a contrast enhancing lesion, or hypoperfusion in a chronic lesion with axonal loss.

As discussed above, endothelial cell dysfunction occurs very early during the inflammatory cascade, and we observe hyperperfusion preceding overt changes in BBB permeability. Most intriguingly, these alterations in perfusion occur even before changes are detectable by any MR imaging technique, such as DTI, MTI, or HMRS. Thus, perfusion MRI may be used as a sensitive indicator of inflammatory activity. Little is known about the cause of early perfusion changes. Invading immune cells presumably stimulate the brain vascular endothelial cells. Additionally, histological studies point to endothelial abnormalities in MS patients. Future investigations of vasoactive components and the interaction of invading immune cells with the resident CNS milieu will increase the knowledge on the underlying disease process. Technical advances made in vivo perfusion imaging feasible even in a routine clinical setting, adding only 1 min to the total scan time with standard EPI-sequences. The postprocessing and calculation requires considerable caution to avoid misinterpretation, as explained in Section 2. Appropriate theoretical models for the exact calculation of an AIF and the quantification of absolute perfusion parameters were recently suggested $[18,23]$, but they have not yet been validated in clinical studies. Perfusion imaging is a very powerful method that provides unique information to supplement and improve our understanding of brain inflammation. It is a potential tool for predicting acute lesion formation and might serve as a valuable imaging technique in monitoring the effects of novel treatments.

\section{Acknowledgement}

The authors wish to acknowledge funding from the Institut für MS-Forschung (IFMS)/Gemeinnützige Hertie-Stifung (GHS) and the Bundesministerium für Bildung und Forschung (BMBF) of Germany. The authors thank Andrew Mason for reading the manuscript as a native English speaker and Peter Brunecker for valuable discussion on technical issues.

\section{Corresponding Author}

Frauke Zipp, frauke.zipp@charite.de 


\section{References}

1. Harris JO, Frank JA, Patronas N, McFarlin DE, McFarland HF. Serial gadolinium-enhanced magnetic resonance imaging scans in patients with early, relapsing-remitting multiple sclerosis: implications for clinical trials and natural history. Ann Neurol 1991;29(5):548-55.

2. McFarland HF, Frank JA, Albert PS, Smith ME, Martin R, Harris JO, et al. Using gadolinium-enhanced magnetic resonance imaging lesions to monitor disease activity in multiple sclerosis. Ann Neurol 1992;32 (6):758-66.

3. Filippi M, Rocca MA, Martino G, Horsfield MA, Comi G. Magnetization transfer changes in the normal appearing white matter precede the appearance of enhancing lesions in patients with multiple sclerosis. Ann Neurol 1998;43(6):809-14.

4. Silver NC, Lai M, Symms MR, Barker GJ, McDonald WI, Miller DH. Serial magnetization transfer imaging to characterize the early evolution of new MS lesions. Neurology 1998;51(3):758-64.

5. Wolinsky JS, Narayana PA. Magnetic resonance spectroscopy in multiple sclerosis: window into the diseased brain. Curr Opin Neurol 2002;15(3):247-51.

6. Werring DJ, Brassat D, Droogan AG, Clark CA, Symms MR, Barker GJ, et al. The pathogenesis of lesions and normalappearing white matter changes in multiple sclerosis: a serial diffusion MRI study. Brain 2000;123(Pt 8):1667-76.

7. Warren JB. Nitric oxide and human skin blood flow responses to acetylcholine and ultraviolet light. Faseb $\mathrm{J}$ 1994;8(2):247-51.

8. Moller K, Strauss GI, Qvist J, Fonsmark L, Knudsen GM, Larsen FS, et al. Cerebral blood flow and oxidative metabolism during human endotoxemia. J Cereb Blood Flow Metab 2002;22(10): 1262-70.

9. Perretti M, Ahluwalia A. The microcirculation and inflammation: site of action for glucocorticoids. Microcirculation 2000;7(3):147-61.

10. Johnson NA, Jahng GH, WeinerMW, Miller BL, Chui HC, Jagust WJ, et al. Pattern of cerebral hypoperfusion in Alzheimer disease and mild cognitive impairment measured with arterial spin-labeling MR imaging: initial experience. Radiology 2005;234(3):851-9.

11. Masdeu JC, Zubieta JL, Arbizu J. Neuroimaging as a marker of the onset and progression of Alzheimer's disease. J Neurol Sci 2005;236 (1-2):55-64.

12. Wuerfel J, Bellmann-Strobl $J$, Brunecker $P$, Aktas $O$, McFarland $H$, Villringer $A$, et al. Changes in cerebral perfusion precede plaque formation in multiple sclerosis: a longitudinal perfusion MRI study. Brain 2004;127(Pt 1):1119.

13. Rashid W, Parkes LM, Ingle GT, Chard DT, Toosy AT, Altmann DR, et al. Abnormalities of cerebral perfusion in multiple sclerosis. J Neurol Neurosurg Psychiatry 2004;75(9):1288-93.

14. Keston $P$, Murray $A D$, Jackson $A$. Cerebral perfusion imaging using contrast-enhanced MRI. Clin Radiol 2003;58(7):505-13.

15. Morse OC, Singer JR. Blood velocity measurements in intact subjects. Science 1970;170(956):440-1.

16. Villringer A, Rosen BR, Belliveau JW, Ackerman JL, Lauffer $\mathrm{RB}$, Buxton RB, et al. Dynamic imaging with lanthanide chelates in normal brain: contrast due to magnetic susceptibility effects. Magn Reson Med 1988;6(2):164-74.

17. Calamante F, Thomas DL, Pell GS, Wiersma J, Turner R. Measuring cerebral blood flow using magnetic resonance imaging techniques. J Cereb Blood Flow Metab 1999; 19(7):701-35.
18. Ostergaard L. Principles of cerebral perfusion imaging by bolus tracking. J Magn Reson Imaging 2005;22(6):710-7.

19. Meier $\mathrm{P}$, Zierler $\mathrm{KL}$. On the theory of the indicator-dilution method for measurement of blood flow and volume. J Appl Physiol 1954;6 (12):731-44

20. Firbank MJ, Coulthard A, Harrison RM, Williams ED. Partial volume effects in MRI studies of multiple sclerosis. Magn Reson Imaging 1999;17(4):593-601.

21. van Osch MJ, Vonken EJ, Wu O, Viergever MA, van der Grond J, Bakker CJ. Model of the human vasculature for studying the influence of contrast injection speed on cerebral perfusion MRI. Magn Reson Med 2003;50(3):61422.

22. Mottet I, Quast MJ, Dewitt DS, Hillman GR, Wei J, Uhrbrock $\mathrm{DH}$, et al. NG-nitro-L-arginine methyl ester modifies the input function measured by dynamic susceptibility contrast magnetic resonance imaging. J Cereb Blood Flow Metab 1997;17(7): 791-800.

23. Conturo TE, Akbudak E, Kotys MS, Chen ML, Chun SJ, Hsu $\mathrm{RM}$, et al. Arterial input functions for dynamic susceptibility contrast MRI: requirements and signal options. J Magn Reson Imaging 2005;22(6):697-703.

24. Rosen BR, Belliveau JW, Aronen HJ, Kennedy D, Buchbinder BR, Fischman A, et al. Susceptibility contrast imaging of cerebral blood volume: human experience. Magn Reson Med 1991;22(2):293-9 [discussion 300-3]

25. Ostergaard L, Sorensen AG, Kwong KK, Weisskoff RM Gyldensted C, Rosen BR. High resolution measurement of cerebral blood flow using intravascular tracer bolus passages. Part II: Experimental comparison and preliminary results. Magn Reson Med 1996;36 (5):726-36.

26. Wirestam R,Andersson L,Ostergaard L,Bolling M,Aunola JP Lindgren A, et al. Assessment of regional cerebral blood flow by dynamic susceptibility contrast MRI using different deconvolution techniques. Magn Reson Med 2000;43(5):691-700.

27. Perkio J, Aronen HJ, Kangasmaki A, Liu Y, Karonen J, Savolainen $\mathrm{S}$, et al. Evaluation of four postprocessing methods for determination of cerebral blood volume and mean transit time by dynamic susceptibility contrast imaging. Magn Reson Med 2002;47(5):973-81.

28. Calamante F, Morup M, Hansen LK. Defining a local arterial input function for perfusion MRI using independent component analysis. Magn Reson Med 2004;52(4):789-97.

29. Calamante F, Gadian DG, Connelly A. Quantification of perfusion using bolus tracking magnetic resonance imaging in stroke: assumptions, limitations, and potential implications for clinical use. Stroke 2002;33(4):1146-51.

30. Haselhorst R, Kappos L, Bilecen D, Scheffler K, Mori D, Radu EW, et al. Dynamic susceptibility contrast MR imaging of plaque development in multiple sclerosis: application of an extended blood-brain barrier leakage correction. J Magn Reson Imaging 2000;11(5):495-505

31. Swank RL, Roth JG, Woody Jr DC. Cerebral blood flow and red cell delivery in normal subjects and in multiple sclerosis. Neurol Res 1983;5(1):37-59.

32. Brooks DJ, Beaney RP, Lammertsma AA, Leenders $\mathrm{KL}$, Horlock PL, Kensett MJ, et al. Quantitative measurement of blood-brain barrier permeability using rubidium-82 and positron emission tomography. J Cereb Blood Flow Metab 1984;4(4):535-45.

33. Lycke J, Wikkelso C, Bergh AC, Jacobsson L, Andersen O Regional cerebral blood flow in multiple sclerosis measured by single photon emission tomography with technetium-99m hexamethylpropyleneamine oxime. Eur Neurol 1993; 33(2):163-7. 
34. Juhler $M$, Paulson OB. Regional cerebral blood flow in acute experimental allergic encephalomyelitis. Brain Res 1986;363(2):272-8.

35. Ge Y, Law M, Johnson G, Herbert J, Babb JS, Mannon LJ, et al. Dynamic susceptibility contrast perfusion MR imaging of multiple sclerosis lesions: characterizing hemodynamic impairment and inflammatory activity. AJNR Am J Neuroradiol 2005;26(6):1539-47.

36. Broom KA, Anthony DC, Blamire AM, Waters S, Styles $P$, Perry $\mathrm{VH}$, et al. MRI reveals that early changes in cerebral blood volume precede blood-brain barrier breakdown and overt pathology in MS-like lesions in rat brain. J Cereb Blood Flow Metab 2005;25(2):204-16.

37. Prineas J. Pathology of the early lesion in multiple sclerosis. Hum Pathol 1975;6(5):531-54.

38. Lucchinetti CF, Brueck W, Rodriguez $M$, Lassmann $H$. Multiple sclerosis: lessons from neuropathology. Semin Neurol 1998;18(3):337-49.

39. Tan IL, van Schijndel RA, Pouwels PJ, van Walderveen MA, Reichenbach JR, Manoliu RA, et al. MR venography of multiple sclerosis. AJNR Am J Neuroradiol 2000;21(6):1039-42.

40. Gareau PJ, Wymore AC, Cofer GP, Johnson GA. Imaging inflammation: direct visualization of perivascular cuffing in EAE by magnetic resonance microscopy. J Magn Reson Imaging 2002;16 (1):28-36.

41. Greter M, Heppner FL, Lemos MP, Odermatt BM, Goebels $\mathrm{N}$, Laufer $\mathrm{T}$, et al. Dendritic cells permit immune invasion of the CNS in an animal model of multiple sclerosis. Nat Med 2005;11(3):328-34.

42. Kostyk SK, Kowall NW, Hauser SL. Substance P immunoreactive astrocytes are present in multiple sclerosis plaques. Brain Res 1989;504(2):284-8.

43. Hartung $T$, Sauer A, Wendel A. Testing of immunomodulatory properties in vitro. Dev Biol Stand 1996;86:85-96.

44. Blamire AM, Anthony DC, Rajagopalan B, Sibson NR, Perry $\mathrm{VH}$, Styles P. Interleukin-1beta-induced changes in bloodbrain barrier permeability, apparent diffusion coefficient, and cerebral blood volume in the rat brain: a magnetic resonance study. J Neurosci 2000;20 (21):8153-9.

45. Sibson NR, Blamire AM, Perry VH, Gauldie J, Styles P, Anthony DC. TNF-alpha reduces cerebral blood volume and disrupts tissue homeostasis via an endothelin- and TNFR2dependent pathway. Brain 2002;125(Pt 11):2446-59.

46. Rosenkranz-Weiss $P$, Sessa WC, Milstien S, Kaufman S, Watson CA, Pober JS. Regulation of nitric oxide synthesis by proinflammatory cytokines in human umbilical vein endothelial cells. Elevations in tetrahydrobiopterin levels enhance endothelial nitric oxide synthase specific activity. J Clin Invest 1994;93(5):2236-43.

47. Yoshizumi M, Perrella MA, Burnett Jr JC, Lee ME. Tumor necrosis factor downregulates an endothelial nitric oxide synthase mRNA by shortening its half-life. Circ Res 1993;73(1):205-9.

48. Kim F, Gallis B, Corson MA. TNF-alpha inhibits flow and insulin signaling leading to NO production in aortic endothelial cells. Am J Physiol Cell Physiol 2001;280(5):C1057-65.

49. Ding $M$, Zhang $M$, Wong JL, Rogers NE, Ignarro $L J$, Voskuhl RR. Antisense knockdown of inducible nitric oxide synthase inhibits induction of experimental autoimmune encephalomyelitis in $\mathrm{SJL} / \mathrm{J}$ mice. $\mathrm{J}$ Immunol 1998;160(6):2560-4.

50. Hooper DC, Bagasra O, Marini JC, Zborek A, Ohnishi ST, Kean $R$, et al. Prevention of experimental allergic encephalomyelitis by targeting nitric oxide and peroxynitrite: implications for the treatment of multiple sclerosis. Proc Nat Acad Sci U S A 1997;94(6):2528-33.

51. Bo L, Dawson TM, Wesselingh S, Mork S, Choi S, Kong PA, et al. Induction of nitric oxide synthase in demyelinating regions of multiple sclerosis brains. Ann Neurol 1994; 36(5):778-86.

52. Rodriguez-Sainz Mdel C, Sanchez-Ramon S, de Andres C, Rodriguez- Mahou M, Munoz-Fernandez MA. Th1/Th2 cytokine balance and nitric oxide in cerebrospinal fluid and serum from patients with multiple sclerosis. Eur Cytokine Netw 2002;13(1):110-4.

53. Mulligan SJ, MacVicar BA. Calciumtransients in astrocyte endfeet cause cerebrovascular constrictions. Nature 2004; 431(7005):195-9.

54. Claudio L, Raine CS, Brosnan CF. Evidence of persistent blood-brain barrier abnormalities in chronic-progressive multiple sclerosis. Acta Neuropathol (Berl) 1995;90(3):22838.

55. Wakefield AJ, More LJ, Difford J, McLaughlin JE. Immunohistochemical study of vascular injury in acute multiple sclerosis. J Clin Pathol 1994;47(2):129-33.

56. Vos CM, Geurts JJ, Montagne L, van Haastert ES, Bo L, van der Valk $\mathrm{P}$, et al. Blood-brain barrier alterations in both focal and diffuse abnormalities on postmortem MRI in multiple sclerosis. Neurobiol Dis 2005;20(3):953-60.

57. Plumb J, McQuaid S, Mirakhur M, Kirk J. Abnorma endothelial tight junctions in active lesions and normalappearing white matter in multiple sclerosis. Brain Pathol 2002;12(2):154-69. $20 \mathrm{~J}$. Wuerfel et al. / Journal of the Neurological Sciences 259 (2007) 16-20. 